PROCEEDINGS OF THE

AMERICAN MATHEMATICAL SOCIETY

Volume 133, Number 5, Pages 1485-1490

S 0002-9939(04)07674-9

Article electronically published on December 6, 2004

\title{
ON A CONJECTURE OF D. STYER REGARDING UNIVALENT GEOMETRIC AND ANNULAR STARLIKE FUNCTIONS
}

\author{
D. BSHOUTY AND A. LYZZAIK
}

(Communicated by Juha M. Heinonen)

\begin{abstract}
The aim of this paper is two-fold. First, to give a direct proof for the already established result of Styer which states that a univalent geometrically starlike function $f$ is a univalent annular starlike function if $f$ is bounded. Second, to show that the boundedness condition of $f$ is necessary, thus disproving a conjecture of Styer.
\end{abstract}

\section{INTRODUCTION}

A region $\Omega$ of the complex plane $\mathbb{C}$ is called starlike with respect to $w_{0} \in \Omega$ if for every point $w \in \Omega$ the closed line segment $\left[w_{0}, w\right]=\left\{(1-t) w_{0}+t w: 0 \leq t \leq 1\right\}$ lies in $\Omega$; in this case $w_{0}$ is called a star center point of $\Omega$. It is well known that the set of star center points of a region $\Omega$, if nonempty, is convex. A univalent function $f$ of the open unit disc $\mathbb{D}$ is said to be geometrically starlike with respect to $w_{0}$ if $f(\mathbb{D})$ is starlike with respect to $w_{0}$. Denote by $S_{g}\left(w_{0}\right)$ the class of all such functions. It is well known that a univalent function $f$ of $\mathbb{D}$ satisfying $f(0)=w_{0}$ is geometrically starlike with respect to $w_{0}$ if and only if $\Re\left\{z f^{\prime}(z) /\left(f(z)-w_{0}\right)\right\}>0$ in $\mathbb{D}$; the condition that $f(0)=w_{0}$ is necessary. Designate by $S_{a}\left(w_{0}\right)$ the class of univalent functions $f$ of $\mathbb{D}$ for which $\Re\left\{z f^{\prime}(z) /\left(f(z)-w_{0}\right)\right\}>0$ when $\rho<|z|<1$ for some $0<\rho<1$; this is the class of annular starlike functions with respect to $w_{0}$. The terminology "geometric starlike" and "annular starlike" is due to Hummel [4].

It is immediate that $S_{a}\left(w_{0}\right)$ is a subset of $S_{g}\left(w_{0}\right)$. Nonetheless the fact that the set-inclusion is proper is not immediate. Individual examples and subclasses of functions $f \in S_{g}\left(w_{0}\right) \backslash S_{a}\left(w_{0}\right)$ were given consecutively by Bender [1], Hengartner and Schober [3], and Goodman and Saff [2. Also, Styer [5] recently demonstrated in a noncomputational way a broad class of functions $f \in S_{g}\left(w_{0}\right) \backslash S_{a}\left(w_{0}\right)$. Furthermore, he proved, using a result about geometrically starlike functions [6], the following result.

Theorem 1. If $f \in S_{g}\left(w_{0}\right)$ is bounded, and if $w_{0}$ is an interior point of the set of star center points of $f(\mathbb{D})$, then $f \in S_{a}\left(w_{0}\right)$.

Following this, Styer [5] made the following conjecture.

Conjecture 1. Theorem 1 holds for unbounded functions $f \in S_{g}\left(w_{0}\right)$.

Received by the editors September 30, 2003 and, in revised form, February 4, 2004.

2000 Mathematics Subject Classification. Primary 30C45.

Key words and phrases. Univalent functions, starlike functions.

The first author thanks the Promotion of Research Fund at the Technion for its support.

(C)2004 American Mathematical Society

Reverts to public domain 28 years from publication 
The purpose of this paper is two-fold. First we establish the following analytic characterization for bounded univalent functions $f$ of $\mathbb{D}$ for which the set of star center points of $f(\mathbb{D})$ has a nonempty interior.

Theorem 2. Let $f$ be a bounded univalent function of $\mathbb{D}$, and let $f\left(z_{0}\right)=w_{0}$ for $z_{0} \in \mathbb{D}$. A necessary and sufficient condition for $w_{0}$ to be an interior point of the set of star center points of $f(\mathbb{D})$ is that there exists a positive $\alpha$ such that

$$
\left|\arg \left\{\Psi\left(z, z_{0}\right) z \frac{f^{\prime}(z)}{f(z)-w_{0}}\right\}\right|<\pi / 2-\alpha, \quad z \in \mathbb{D},
$$

where $\Psi\left(z, z_{0}\right)=\left(z-z_{0}\right)\left(1-\bar{z}_{0} z\right) / z, \Psi(z, 0) \equiv 1$, and $\arg$ is the principal argument function.

The result yields at once Theorem 1 (without using Styer's result [6].) The second purpose of the paper is to disprove Conjecture 1

\section{Proof of Theorem 2}

For the necessity proof, let $\Delta$ be a compact disc centered at $w_{0}$ and lying in the set of star center points of $f(\mathbb{D})$, and let $K$ be a compact subset of $f(\mathbb{D})$ containing $\Delta$ in its interior. For $a \in \partial f(\mathbb{D})$, let $b$ and $c$ be the points of $\partial \Delta$ for which the line segments $[b, a]$ and $[c, a]$ are tangent to $\Delta$ and lie, except for $a$, on the left- and right-hand side of the line segment $\left[w_{0}, a\right]$ respectively. Denote by $\Delta_{a}$ the region bounded by $[b, a],[c, a]$, and the major arc of $\partial \Delta$ ending in $b$ and $c$. Note that $\overline{\Delta_{a}} \backslash\{a\}$ lies in $f(\mathbb{D})$ for all $a \in \partial \Delta$.

It is easily seen that the collection $\left\{\Delta_{a}: a \in \partial f(\mathbb{D})\right\}$ is an open covering of $K$, and that by compactness $K \subset \Delta_{a_{1}} \cup \Delta_{a_{2}} \cup \cdots \cup \Delta_{a_{r}}$ for some $a_{1}, a_{2}, \cdots, a_{r}$ in $\partial f(\mathbb{D})$. Observe that no $\Delta_{a_{i}}$ is contained in $\bigcup_{j \neq i} \Delta_{a_{j}}$ and that the open-closed segments $\left(w_{0}, a_{i}\right]$ are mutually disjoint. Re-label the points $a_{i}$ so that each $0 \leq \arg \left(a_{i}-w_{0}\right)<$ $2 \pi$ and $\arg \left(a_{i}-w_{0}\right)$ strictly increases with $i$. With $a=a_{i}$, let $b=b_{i}$ and $c=c_{i}$. Note that for $1 \leq i \leq r$, the points $c_{i}, c_{i+1}, b_{i}, b_{i+1}$, with $c_{1}=c_{r+1}$ and $b_{1}=b_{r+1}$, are distinct and they appear on $\partial \Delta$ as listed when it is positively traversed. Denote by $d_{i}$ the point of intersection of the open segments $\left(a_{i}, b_{i}\right)$ and $\left(a_{i+1}, c_{i+1}\right)$, with $a_{1}=a_{i+1}$. Let $G=\bigcup_{i=1}^{r} \Delta_{a_{i}}$. Then $G$ is a simply connected (2r)-polygonal region with vertices $a_{i}$ and $d_{i}, K \subset G$, and $G$ admits $\Delta$ as a subset of its set of star center points. Furthermore, the size of each of the interior and exterior angles of $\partial G$ at the vertices $a_{i}$ and $d_{i}$ is less than $\pi$. Round the corners of $G$ at each $a_{i}$ from inside of $G$ and at each $d_{i}$ from outside of $G$ in a manner that preserves the properties of $G$; denote, for convenience, the resulting region by $G$. Let $F$ be the Riemann mapping from $\mathbb{D}$ onto $G$ with $F\left(z_{0}\right)=w_{0}$ and $\arg F^{\prime}\left(z_{0}\right)=\arg f^{\prime}\left(z_{0}\right)$.

With $a \in \partial f(\mathbb{D})$, let $2 \alpha$ be a positive lower bound of the size of the angles $\angle b a c$. Evidently $0<\alpha<\pi / 2$. Henceforth, assume $a \in \partial G$, and let $b, c$ and $\Delta_{a}$ be as defined above. Because $G \subset f(\mathbb{D})$, the size of $\angle b a c$ is at least $2 \alpha$ for all $a \in \partial G$. Observe that the angle between the outward normal vector of $\partial G$ at $a$ and the vector from $w_{0}$ to $a$ is at most $\pi / 2-\alpha$. Since $\partial G$ is smooth, this yields $\left|\arg \left[z F^{\prime}(z) /\left(F(z)-w_{0}\right)\right]\right| \leq \pi / 2-\alpha$ for $z \in \partial \mathbb{D}$. Furthermore, by [8] Theorem 3.2], there exists $0<\rho<1$ such that $\arg \left[z F^{\prime}(z) /\left(F(z)-w_{0}\right)\right]$ is continuous for $\rho \leq$ $|z| \leq 1$. Since $\arg \Psi\left(z, z_{0}\right) \equiv 0$ for $|z|=1, F$ is analytic in $\mathbb{D}$, and $\Psi\left(z, z_{0}\right) z F^{\prime}(z) /$ $\left(F(z)-w_{0}\right)$ is a continuous and nonvanishing function in the closed unit disc, inequality (1) holds for $F$ by the maximum principle. 
To complete the proof, exhaust $\Omega$ by an increasing sequence of compact subsets $K_{n}$ each containing $\Delta$ in its interior, and let $F_{n}$ be the function $F$ associated with $K_{n}$. Because $F_{n}(\mathbb{D}) \rightarrow f(\mathbb{D})$ as $n \rightarrow \infty, F_{n}\left(z_{0}\right)=w_{0}$ and $\arg F_{n}^{\prime}\left(z_{0}\right)=\arg f^{\prime}\left(z_{0}\right)$, the Carathéodory kernel theorem yields the local uniform convergence $F_{n} \rightarrow f$ as $n \rightarrow \infty$. We conclude that inequality (1) holds for $f$ since it holds for each $F_{n}$. This ends the proof of necessity.

For another proof of necessity, $f \in S_{g}\left(w_{0}\right)$ means that the function

$$
g(\zeta)=f\left(\frac{\zeta+z_{0}}{1+\bar{z}_{0} \zeta}\right)-w_{0}
$$

is a univalent starlike function that satisfies $g(0)=0$. That is, $\Re\left[\zeta g^{\prime}(\zeta) / g(\zeta)\right]>0$ in $\mathbb{D}$, or, by letting $z=\left(\zeta-z_{0}\right) /\left(1-\bar{z}_{0} \zeta\right)$,

$$
\Re\left\{\Psi\left(z, z_{0}\right) z \frac{f^{\prime}(z)}{f(z)-w_{0}}\right\}>0, \quad z \in \mathbb{D},
$$

or, since $\arg \Psi\left(z, z_{0}\right) \equiv 0$ for $|z|=1$, for $\epsilon>0$ there exists $\rho, 0<\rho<1$, such that

$$
\left|\arg z f^{\prime}(z) /\left(f(z)-w_{0}\right)\right|<\pi / 2+\epsilon, \quad \rho<|z|<1 .
$$

The rest of the proof now proceeds as in the proof of Styer [5. Theorem 1] without using his result about geometrically starlike functions [6]. This ends the second necessity proof.

For the sufficiency proof, inequality (1) yields $\rho, 0<\rho<1$, such that

$$
\left|\arg \frac{z f^{\prime}(z)}{f(z)-w_{0}}\right|<\pi / 2-\alpha / 2, \quad \rho<|z|<1 .
$$

Note that $\arg \left\{z f^{\prime}(z) /(f(z)-w)\right\}$ is a continuous function in $(z, w)$ for values $z$, $\rho<|z|<1$, and $w$ sufficiently close to $w_{0}$. Fix $\sigma, \rho<\sigma<1$. By a compactness argument, there exists a compact disc $\Delta$ centered at $w_{0}$ such that

$$
\left|\arg \frac{z f^{\prime}(z)}{f(z)-w}\right|<\pi / 2-\alpha / 3, \quad|z|=\sigma, \quad w \in \Delta .
$$

Thus, for $|z|=\sigma$ and $w \in \Delta, \Re\left\{z f^{\prime}(z) /(f(z)-w)\right\}>0$ and the closed-open line segment $[w, f(z))$ lies in the image set of $f$ as restricted to the open disc $|z|<\rho$. It follows that for every $z, \rho<|z|<1$, the closed line segment $[w, f(z)]$ lies in $f(\mathbb{D})$. It is immediate that this property extends for all $z \in \mathbb{D}$. This ends the sufficiency proof and completes the proof of Theorem 2

\section{Disproof of Conjecture 1}

In this section we disprove Conjecture 1 [5].

For a fixed $\alpha, 1<\alpha<2$, let

$$
f(z)=\left(\frac{1+z}{1-z}\right)^{\alpha}, \quad z \in \mathbb{D} .
$$

This is a univalent (close-to-convex) function of $\mathbb{D}$ whose image set $f(\mathbb{D})$ is the wedge $\{w:|\arg w|<\alpha \pi / 2\}$ for which the set of star center points is $E=\{w$ : $|\arg w| \leq(2-\alpha) \pi / 2\}$. With $f(\bar{z})=\overline{f(z)}$, the function preserves symmetry about the real axis. Denote by $\Gamma_{r}$ the curve parameterized by $f\left(r e^{i \theta}\right), 0 \leq \theta \leq 2 \pi$. Then $\Gamma_{r}$ is a Jordan curve that is symmetric about the real axis with its upper-half (the part that lies in the closed upper-half plane) starting and terminating at the points $((1+r) /(1-r))^{\alpha}$ and $((1-r) /(1+r))^{\alpha}$ respectively. 
Henceforth, we show that $f \notin S_{a}(w)$ for every $w$ in some real open interval $(0, b)$, which obviously lies in the interior of $E$.

Direct computation yields

$$
\begin{aligned}
\Re\left\{1+z \frac{f^{\prime \prime}(z)}{f^{\prime}(z)}\right\} & =\Re\left\{\frac{1+2 \alpha z+z^{2}}{1-z^{2}}\right\} \\
& =\frac{1-|z|^{2}}{\left|1-z^{2}\right|^{2}} \Re\left(1+2 \alpha z+|z|^{2}\right) .
\end{aligned}
$$

By letting $R(r, \theta)=\Re\left\{1+z f^{\prime \prime}(z) / f^{\prime}(z)\right\}$ for $z=r e^{i \theta}$, we obtain

$$
R(r, \theta)=\frac{1-r^{2}}{\left|1-r^{2} e^{2 i \theta}\right|^{2}}\left(1+2 \alpha r \cos \theta+r^{2}\right) .
$$

Note that $\left(1+r^{2}\right) /(2 \alpha r)<1$ if and only if $r$ belongs to the open interval $I=(\alpha-$ $\left.\sqrt{\alpha^{2}-1}, 1\right)$. Fix $r \in I$, and let $\Theta_{r}=\cos ^{-1}\left(-\left(1+r^{2}\right) /(2 \alpha r)\right)$, where $\pi / 2<\Theta_{r}<\pi$. The function $R(r, \theta)$ is positive if $-\Theta_{r}<\theta<\Theta_{r}$, negative if $\Theta_{r}<\theta<2 \pi+\Theta_{r}$, and zero if $\theta= \pm \Theta_{r}$. Let $z_{r}=r e^{i \Theta_{r}}$. Since $R(r, \theta)$ is the rate of change of the argument of the tangent vector to $\Gamma_{r}$ at $r e^{i \theta}, \Gamma_{r}$ has exactly two points of inflection, namely the points $f\left(z_{r}\right)$ and $f\left(\overline{z_{r}}\right)$. Denote by $L_{r}$ the tangent line to $\Gamma_{r}$ at $f\left(z_{r}\right)$, and let $b_{r}$ be the point of intersection, if it exists, of this line with the real axis. (In fact, because of symmetry, the tangent lines to $\Gamma_{r}$ at $f\left(z_{r}\right)$ and $f\left(\overline{z_{r}}\right)$ are symmetric about the real axis and meet the real axis at the same point $b_{r}$, if it exists.)

Note that a parametric equation of $L_{r}$ is given by $f\left(z_{r}\right)+i t z_{r} f^{\prime}\left(z_{r}\right),-\infty<t<$ $\infty$, which meets the real axis if $\Im f\left(z_{r}\right)+t \Re\left(z_{r} f^{\prime}\left(z_{r}\right)\right)=0$, or if $t$ assumes the value $t_{r}=-\Re\left(z_{r} f^{\prime}\left(z_{r}\right)\right) / \Im f\left(z_{r}\right)$. Thus $b_{r}$ exists and

$$
b_{r}=f\left(z_{r}\right)-i z_{r} f^{\prime}\left(z_{r}\right) \frac{\Re\left(z_{r} f^{\prime}\left(z_{r}\right)\right)}{\Im f\left(z_{r}\right)} .
$$

Being real,

$$
\begin{aligned}
b_{r} & =\Re f\left(z_{r}\right)+\frac{\Re\left(z_{r} f^{\prime}\left(z_{r}\right)\right) \Im\left(z_{r} f^{\prime}\left(z_{r}\right)\right)}{\Im f\left(z_{r}\right)} \\
& =\frac{\Re f\left(z_{r}\right) \Im f\left(z_{r}\right)+\Re\left(z_{r} f^{\prime}\left(z_{r}\right)\right) \Im\left(z_{r} f^{\prime}\left(z_{r}\right)\right)}{\Im f\left(z_{r}\right)} \\
& =\frac{\Im\left[f(z)^{2}+\left(z_{r} f^{\prime}\left(z_{r}\right)^{2}\right)\right]}{2 \Im f\left(z_{r}\right)} \\
& =\frac{\Im\left[f(z)^{2}\left\{1+\left(z_{r} f^{\prime}\left(z_{r}\right) / f\left(z_{r}\right)\right)^{2}\right\}\right]}{2 \Im f\left(z_{r}\right)} \\
& =\frac{\Im\left[f(z)^{2}\left\{1+\left(2 \alpha z_{r} /\left(1-z_{r}^{2}\right)\right)^{2}\right\}\right]}{2 \Im f\left(z_{r}\right)} .
\end{aligned}
$$

Note that $\lim _{r \rightarrow 1^{-}} z_{r}=\left(-1+i \sqrt{\alpha^{2}-1}\right) / \alpha$ since $\lim _{r \rightarrow 1^{-}} \Theta_{r}=\cos ^{-1}(-1 / \alpha)$, and consequently,

$$
1+\left(\frac{2 \alpha z_{r}}{\left(1-z_{r}^{2}\right)}\right)^{2} \rightarrow 1+\frac{\alpha^{4}}{1-\alpha^{2}}, \quad \text { as } \quad r \rightarrow 1^{-}
$$




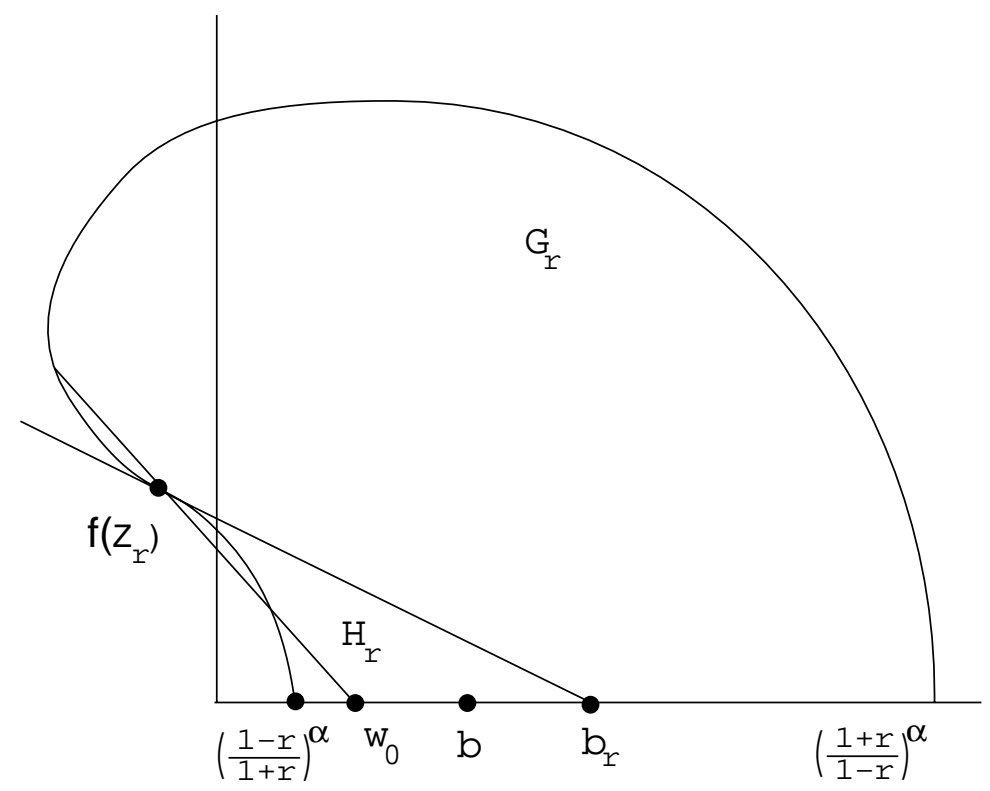

Figure 1. Disproof of Conjecture 1

Furthermore, by setting $\Theta=\cos ^{-1}(1 / \alpha)$,

$$
\begin{aligned}
\frac{\Im\left(f\left(z_{r}\right)^{2}\right)}{2 \Im f\left(z_{r}\right)} & \rightarrow \frac{\Im\left[\left(1+e^{i \Theta}\right)^{2 \alpha} /\left(1-e^{i \Theta}\right)^{2 \alpha}\right]}{2 \Im\left[\left(1+e^{i \Theta}\right)^{\alpha} /\left(1-e^{i \Theta}\right)^{\alpha}\right]} \quad \text { as } r \rightarrow 1^{-} \\
& =[\cot (\Theta / 2)]^{\alpha} \cos (\alpha \pi / 2) \\
& =[(\alpha-1) /(\alpha+1)]^{\alpha / 2} \cos (\alpha \pi / 2) .
\end{aligned}
$$

Thus if $b=\lim _{r \rightarrow 1^{-}} b_{r}$, then, by using equations (3) and (4) in equation (2), we conclude that

$$
b=\left[1+\alpha^{4} /\left(1-\alpha^{2}\right)\right][(\alpha-1) /(\alpha+1)]^{\alpha / 2} \cos (\alpha \pi / 2),
$$

which is obviously positive.

Fix $w_{0} \in(0, b)$; see Figure 1 We show that $f \notin S_{a}\left(w_{0}\right)$. It can be easily verified that $\lim _{r \rightarrow 1^{-}} f(r)=\infty, \lim _{r \rightarrow 1^{-}} f(-r)=0$,

$$
\lim _{r \rightarrow 1^{-}} f\left(z_{r}\right)=e^{i \alpha \pi / 2}\left(\frac{1-\alpha}{1+\alpha}\right)^{\alpha / 2}
$$

lies in the open second quadrant of $\mathbb{C}$, and

$$
\lim _{r \rightarrow 1^{-}} t_{r}=\frac{\alpha^{2}}{\sqrt{\alpha^{2}-1}}
$$

is positive. In view of this, there exists $\rho, 0<\rho<1$, such that for any $r, \rho<r<1$, the points $w_{0}, b_{r}$ and $b$ lie in the interior region of $\Gamma_{r}$ with $b_{r}>w_{0}, f\left(z_{r}\right)$ lies in the open second quadrant of $\mathbb{C}$, and $t_{r}$ is positive. The latter fact implies that the tangent vector to $\Gamma_{r}$ at $f\left(z_{r}\right)$ has the same direction as the vector from $f\left(z_{r}\right)$ to $b_{r}$. Endow this direction to $L_{r}$, and denote by $\delta_{r}$ and $\sigma_{r}$ the subarcs of $\Gamma_{r}$ parameterized by $f\left(r e^{i \theta}\right), 0 \leq \theta \leq \Theta_{r}$, and $f\left(r e^{i \theta}\right), \Theta_{r} \leq \theta \leq \pi$, respectively. By invoking the mapping properties of $f$ on the circle $|z|=r$, the arc $\delta_{r}$ and the line segments 
$\left[f\left(z_{r}\right), b_{r}\right]$ and $\left[b_{r},((1+r) /(1-r))^{\alpha}\right]$ bound a convex Jordan subregion, $G_{r}$, of the upper-half of the interior region of $\Gamma_{r}$; note that $G_{r}$ lies on the left-hand side of $L_{r}$. Also, the $\operatorname{arc} \sigma_{r}$ and the line segments $\left[f\left(z_{r}\right), b_{r}\right]$ and $\left[((1-r) /(1+r))^{\alpha}, b_{r}\right]$ bound a Jordan subregion, $H_{r}$, of the upper-half of the interior region of $\Gamma_{r}$; note also that $H_{r}$ lies on the right-hand side of $L_{r}$. By virtue of the direction of the tangent vector to $\Gamma_{r}$ at $f\left(z_{r}\right)$ and the fact that $((1-r) /(1+r))^{\alpha}<w_{0}<b_{r}$, we conclude that the ray from $w_{0}$ through $f\left(z_{r}\right)$ crosses, consecutively, once each of the interiors of the $\operatorname{arcs} \sigma_{r}$ and $\delta_{r}$ in a manner that yields a cross-cut in $G_{r}$. Therefore, $f \notin S_{a}\left(w_{0}\right)$ for every $w_{0} \in(0, b)$ and Conjecture 1 is false.

\section{REFERENCES}

[1] J. Bender, Some extremal theorems for multivalently star-like functions, Duke Math. J. 29 (1962), 101-106. MR0132171 (24:A2018)

[2] A. W. Goodman and E. B. Saff, On univalent functions convex in one direction, Proc. Amer. Math. Soc. 73 (1979), 183-187. MR0516461 (80e:30006)

[3] W. Hengartner and G. Schober, A remark on level curves for domains convex in one direction, Appl. Anaysis 3 (1973), 101-106. MR0393450 (52:14260)

[4] J. A. Hummel, Multivalent starlike functions, J. Analyse Math. 18 (1967), 133-160. MR 0209461 (35:359)

[5] D. Styer, Geometric and annular starlike functions, Complex Variables 29 (1996), 189-191. MR.1382965 (97b:30021)

[6] D. Styer, An analytic characterization of geometrically sta rlike functions, Michigan Math. J. 23 (1976), 137-139. MR0409792 (53:13544)

[7] Ch. Pommerenke, Univalent functions, Vandenhoeck \& Ruprec ht, Göttingen, 1975. MR 0507768 (58:22526)

[8] Ch. Pommerenke, Boundary behaviour of conformal maps, Springer-Verlag, Berlin, 1992. MR:1217706 (95b:30008)

Department of Mathematics, Technion, Haifa 32000, Israel

E-mail address: daoud@tx.technion.ac.il

Department of Mathematics, American University of Beirut, Beirut, Lebanon

E-mail address: lyzzaik@aub.edu.lb 